\title{
Stage III Fallopian Tube Cancer AJCC v8
}

National Cancer Institute

\section{Source}

National Cancer Institute. Stage III Fallopian Tube Cancer A/CC v8. NCI Thesaurus. Code C139991.

Stage III includes: IIIA1: T1/2, N1, M0; IIIA2: T3a, N0/N1, M0; IIIB: T3b, N0/N1, M0; IIIC: T3c, N0/N1, M0. T1: Fallopian tube cancer with tumor limited to fallopian tube(s). T2: Fallopian tube cancer with tumor involving fallopian tubes with pelvic extension below pelvic brim. T3a: Fallopian tube cancer with microscopic extrapelvic (above the pelvic brim) peritoneal involvement with or without positive retroperitoneal lymph nodes. T3b: Fallopian tube cancer with macroscopic peritoneal metastasis beyond pelvis $2 \mathrm{~cm}$ or less in greatest dimension with or without metastasis to the retroperitoneal lymph nodes. T3c: Fallopian tube cancer with macroscopic peritoneal metastasis beyond the pelvis more than $2 \mathrm{~cm}$ in greatest dimension with or without metastasis to the retroperitoneal lymph nodes (includes extension of tumor to capsule of liver and spleen without parenchymal involvement of either organ). N0: No regional lymph node metastasis. N1: Positive retroperitoneal lymph nodes only (histologically confirmed). M0: No distant metastasis. (AJCC 8th Ed.) 